\title{
Correction
}

\section{Correction: AKT Inhibitors Promote Cell Death in Cervical Cancer through Disruption of mTOR Signaling and Glucose Uptake}

\section{The PLOS ONE Staff}

The sixth author's name is spelled incorrectly. The correct name is: Janet L. Rader.

\section{Reference}

1. Rashmi R, DeSelm C, Helms C, Bowcock A, Rogers BE, et al. (2014) AKT Inhibitors Promote Cell Death in Cervical Cancer through Disruption of mTOR Signaling and Glucose Uptake. PLoS ONE 9(4): e92948. doi:10.1371/ journal.pone.0092948 